\title{
II dovere di solidarietà. Spunti di riflessione su Diritto Pubblico e Dottrina Sociale della Chiesa
}

\author{
The Duty of Solidarity. Considerations around Public Law \\ and Social Doctrine of the Church
}

\author{
Agata C. Amato Mangiameli \\ Universitá degli Studi di Roma «Tor Vergata» (Italia) \\ amatomangiameli@gmail.com
}

\begin{abstract}
Riassunto: La dialettica tra cittadino e straniero illumina la profonda distanza che separa il Diritto Pubblico dalla Dottrina Sociale della Chiesa. Per il primo, l'identità del cittadino e l'identità del noi politico sono regolate dalle leggi che governano la distribuzione dell'appartenenza, per la seconda, invece, nessuno è straniero, e la Chiesa non è straniera a nessun uomo e in nessun luogo. Dobbiamo semplicemente accontentarci di questa distinzione, e con essa sopportare le separazioni identitarie di gruppi, di popoli e nazioni che essa implica e suppone, oppure lo Stato e il diritto pubblico possono assumere un nuovo ruolo e guadagnare un nuovo sguardo sull'altro-a/ sugli-sulle altri-e? Cercando di fornire una valida risposta a questa domanda, l'Autore ricostruisce la categoria della cittadinanza sotto il segno del dovere, identificando nel termine dovere l'elemento che può unire libertà-dignitàuguaglianza e bene comune. Mantenendo intrecciate le dimensioni del particolare e dell'universale, possiamo dunque propendere per il definitivo superamento della dialettica tra cittadino e civis mundi: una società coesa, aperta e solidale è possibile solo quando entrambi conoscono e rispettano i propri reciproci doveri.
\end{abstract}

Parole chiave: solidarietà; dottrina sociale della Chiesa; diritto pubblico; doveri universali; cittadinanza.

\begin{abstract}
The dialectic between citizens and a foreigners illuminates the profound distance which divides Public Law from the Social Doctrine of the Church. According to the first, the identity of the citizen and the identity of the community are ruled by the laws which rule the belonging. According to the second, no one is a foreigner, and the Church is not foreign to any man. In no place. Should we be content with such distinction and endure the separation of groups, peoples and nations that it implies and supposes? Or should the State and the public law gain a new perspective on the problem? Trying to provide a valid answer to this question, the Author reconstructs the category of citizenship under the sign of duty, identifying in the term «duty» the only concept which could link together freedom, dignity, equality and common good. Keeping universal and particular intertwined, we can therefore propose the definitive overcoming of the ancient dialectic between citizen and civis mundi. Because a cohesive, open and caring society is possible if only both of them know and respect their own duties.
\end{abstract}

Keywords: solidarity; social doctrine of the Church; public law; universal duties; citizenship.

\section{PRemessa}

1 tema è complesso ed è decisamente ampio. Tra i diversi argomenti, importante e di grande attualità è la dialettica cittadino/straniero, una dialettica questa che rende immediatamente evidente la diversità di ambiti e di prospettive, la profonda distanza del diritto pubblico e della dottrina sociale della Chiesa. 
Per il primo, l'identità del cittadino e l'identità del noi politico sono segnate dai principi interni ed esterni che governano la distribuzione dell'appartenenza, così che nello Stato il carattere chiuso dell'appartenenza può consolidare l'esclusione, e invece per la seconda ciò non si dà: nella Chiesa nessuno è straniero, e la Chiesa non è straniera a nessun uomo e in nessun luogo ${ }^{1}$.

$\mathrm{Ci}$ si deve semplicemente accontentare di simile distinzione, rassegnarsi alla distanza, e con essa sopportare le separazioni identitarie di gruppi, di popoli, di nazioni, oppure lo Stato e il diritto pubblico possono assumere un nuovo ruolo e un nuovo sguardo sull'altro-a/sugli-sulle altri-e? C'è ragione di pensare che, non solo, una tale possibilità esista, ma che essa sia anzi necessaria per la ricostruzione di un tessuto politico istituzionale e sociale che non alimenti meccanismi di esclusione e che non sia animato da mere logiche di potere. E questo vale sia all'interno dei confini nazionali, sia all'esterno. All'interno, perché nel contesto di una società internazionale complessa, con un'ampia gamma di organizzazioni regionali e globali che trascendono e mediano i confini nazionali, il ruolo dello Stato non può non mutare, quantomeno per fronteggiare quelle logiche e quelle dinamiche proprie della globalizzazione che sono senz'altro da valutare negativamente. All'esterno, perché con l'aumento dell'interdipendenza globale - e più in particolare con la rapida espansione dei legami intergovernativi e transnazionali - emerge la necessità di regolamentare le c.d. zone di nessuno che richiedono un nuovo nomos, quantomeno per fronteggiare le molteplici e diverse alleanze di potere, subentrate alle strutture di potere e alle costruzioni giuridiche di attori nazional-statali che «si chiudono l'una all'altra e si combattono»².

\section{DUE TENTAZIONI RIDUZIONISTE: DIRITTO PRIVATO/DIRITTO PUBBLICO}

Nell'attraversare per così dire il diritto, si incontrano due tentazioni riduzioniste che sono responsabili di una lettura poco attenta sia del diritto sia dello Stato; una lettura che, anzi, non tiene alcun conto del senso e della ricchezza del fenomeno giuridico e che complica a tal punto il rapporto diritto/politica

1 Giovanni PaOlo II, «Migranti irregolari. Messaggio per la giornata mondiale dell'emigrazione», <http://w2.vatican.va/content/john-paul-ii/it/messages/migration/documents/hf_jpii_mes_25071995_undocumented_migrants.html>, 1995 (18 agosto 2017).

2 Sempre attuali le ricostruzioni di BECK, U., Che cos'è la globalizzazione: rischi e prospettive della società planetaria, trad. it., Carocci, Roma, 1999, pp. 193 s. 
da ritenere irrisolvibile proprio la dialettica cittadino/straniero. D'altra parte, tanto questa dialettica così come l'assai spesso aspra contrapposizione che ne consegue sono da sempre note e - seppure con sfumature diverse - praticate, se è vero con Eschilo che la gente forestiera subisce l'esame del tempo. Sull'emigrante, è svelta a scattare la lingua cattiva. La chiacchiera sfreccia, t'infanga!

La prima tentazione riduzionista è quella che considera diritto solo il diritto privato. La ragione è piuttosto semplice e nella sua istanza minima (prius logico rispetto al diritto pubblico) anche condivisibile. Il diritto privato è l'insieme dei rapporti giuridici tra persone giuridicamente uguali, i cui diritti sono riconosciuti e protetti e i cui doveri derivano di regola da un'auto-sottomissione. Qui il protagonista assoluto è il soggetto di diritto: un soggetto che calca la scena mutando di frequente abito, a seconda che i diritti siano prospettati come potestà di volere, come interessi giuridicamente protetti, come interessi pubblici soggettivi o, semplicemente, come situazioni giuridiche soggettive. In ogni caso, si tratta di un soggetto che non coincide e non si identifica sempre con il cittadino, poiché il diritto privato è la disciplina dei rapporti della persona tout court, la quale - come ha affermato $\mathrm{Kant}^{3}$ - costituisce il centro di ogni sistema e di ogni ordinamento giuridico.

La seconda tentazione riduzionista, invece, è quella che considera diritto solo il diritto pubblico o che quantomeno rivendica il primato di quest'ultimo. Anche in questa ipotesi la ragione è piuttosto semplice. Il primato, infatti, spetterebbe al diritto pubblico, dal momento che qualsiasi rapporto di diritto privato non può darsi che nello Stato, vale a dire tra individui che rivestono la qualità di cittadini o di sudditi e che - in quanto tali - riconoscono l'esistenza di un ordine politico sovrastante e preminente rispetto ai loro interessi privati e alle loro finalità particolari.

Del resto, se ci si domanda che cosa sia il diritto pubblico, una prima e semplice definizione suona in tal modo: è l'insieme dei principi giuridici, che regolano lo Stato, i suoi organi e la sua organizzazione, il potere di governo, le funzioni e i servizi. Appartengono allora all'ambito pubblico tutti quei rapporti in cui lo Stato interviene in via ufficiale, così come la determinazione dei diritti e dei doveri dei funzionari pubblici, nonché del loro status e delle loro attribuzioni (competenze). Ma non è tutto, perché il diritto pubblico ricomprende anche tutte quelle azioni che vengono svolte dall'individuo in quanto cittadino (o anche in quanto straniero): ad esempio, chi va a votare, eserci-

3 Fondazione della metafisica dei costumi, trad. it., Laterza, Roma-Bari, 1993. 
tando un diritto-dovere, il contribuente dal quale il fisco reclama l'imposta, il soldato in caserma, l'automobilista con il codice stradale, e così via ${ }^{4}$.

Ambedue le pretese - tra loro ovviamente contraddittorie: diritto è solo il diritto privato/diritto è solo il diritto pubblico - oltre a rivelarsi distanti dai criteri di giustificazione dell'obbligatorietà normativa non tengono conto delle tante nuance, tutte essenziali, proprie del fenomeno giuridico.

Detto molto semplicemente, l'ordine giuridico non si esaurisce né nella dimensione orizzontale dei rapporti di diritto privato, né nella dimensione verticale dei rapporti di diritto pubblico. E ciò è tanto più vero per i sistemi giuridici contemporanei, con le loro dimensioni di alta vincolatività (bard law) e di alta flessibilità (soft law $)^{5}$.

2.1. Nel mondo senza frontiere, il sistema giuridico sempre più è determinato dall'agenda economica e sempre più le sue funzioni (legislativa e giudiziaria) sono per dir così attratte dagli usi del commercio internazionale. Il che significa: crescente privatizzazione e decentramento della produzione giuridica, quindi elaborazione delle regole al di fuori della sfera pubblica; crescente ricorso all'arbitrato quale meccanismo di risoluzione delle controversie, dunque soluzione della disputa al di fuori della mediazione statale ${ }^{6}$. Altrimenti detto, gli operatori economici tendono a scegliere i loro giudici per le loro dispute d'affari e anzi a essere giudici nell'applicazione di un diritto specifico e adatto ormai ai bisogni del commercio internazionale (law shopping). E sempre gli operatori economici hanno bisogno dei cd. mercanti del diritto, pronti a elaborare misure giuridiche adeguate a un mercato in espansione e in grado di risolvere in luogo dei tribunali le controversie medesime. Si assiste così, per un verso, al proliferare di regole mutevoli e instabili: la lex mercatoria non è più «un corpo di misure giuridiche elaborate dalla tradizione del mondo commerciale e che esprime usi e consuetudini; diventa anzi piuttosto il suo contrario, ossia un universo normativo estremamente mobile, soggetto a continue manipolazioni e ritocchi che sono paralleli alla estrema mobilità del mercato». E, per un altro verso, si riscontra un aumento dei soggetti produttori del diritto, «soggetti legislatori sempre più privati, anonimi e invisibili»,

4 Per ulteriori considerazioni vedi il mio «Parti e protagonisti del diritto: privato e pubblico», in Amato Mangiameli, A. C., Arte elo tecnica. Sfide giuridiche, Cedam, Padova, 2012, pp. 79-87.

5 Già evidenziate parecchi anni fa da FerRARI, V., Lineamenti di sociologia del diritto. I. Azione giuridica e sistema normativo, Laterza, Roma-Bari, 1997, p. 320 s.

6 Rinvio al mio Stati post-moderni e diritto dei popoli, Giappichelli, Torino, 2004. 
che, con il loro potere (indipendente) di legislazione, non soltanto mostrano che il diritto non è più eminentemente statale e che non si dà alcuna autorità pienamente autonoma e risolutiva, ma che il diritto (negoziato) può non essere distinto dagli interessi ${ }^{7}$.

2.2. Ma non è solo la globalizzazione economica, che mescola pubblico e privato, a determinare tale frammentazione e a far venir meno la potenza ordinatrice degli Stati. Sono anche i diversi organismi interstatali - e a maggior ragione le istituzioni sovra-statali, risultato di accordi, convenzioni, trattati (tra gli Stati), ed espressione della necessità di individuare nuove forme di cooperazione e regolamentazione - che determinano il pluralismo giuridico e, come si usa ormai dire, il regime internazionale: cioè le norme, i regolamenti, i principi (impliciti ed espliciti), le procedure decisionali, usati in una determinata sfera delle relazioni internazionali.

Più in particolare, l'aumento della cooperazione tra gli Stati, che coinvolge governi, organizzazioni intergovernative, non governative, gruppi di pressione transnazionali, favorisce un modello di regolazione a rete. Il che significa: a) una pluralità di soggetti regolatori, con competenze concorrenti in senso lato; b) un riordino delle fonti, piuttosto che secondo criteri di gerarchia formale, secondo criteri sostanziali, e quindi in base a principi e obiettivi comuni; c) un sistema di procedure di raccordo tra i diversi soggetti regolatori, così che siano ridotti i rischi di scostamenti e alterazioni dovuti al tradursi dei principi comuni in discipline applicative di dettaglio. E se è così, può ben dirsi: «del mitico modello kelseniano, perfettamente rigoroso e deduttivo nel basarsi su un centro unico e supremo cui ricondurre l'intera attività di produzione normativa, è dunque rimasto, in questo scenario di sovranità multiple, ben poco: tramontata l'idea di un centro unitario di produzione delle regole normative, l'odierna norma di riconoscimento va vista, più che in una norma fondamentale, in una pratica giurisdizionale e nei principi che la informano ${ }^{8}$.

7 Non a caso «si istituzionalizza una complessa interazione di soggetti diversi per continue ridefinizioni giuridiche che rispondano meglio a fini di 'acquisizione'». E in un contesto siffatto «è l'istituzione giudiziaria, per la sua attitudine a funzionare come istituzione di raccordo tra casi particolari e istanze generali, tra pubblico e privato, tra locale e globale, che si candida a essere l'istituzione centrale e funzionale rispetto ai percorsi della globalizzazione» (così FERRARESE, M.A., Le istituzioni della globalizzazione. Diritto e diritti nella società transnazionale, Il Mulino, Bologna, 2000, p. 50 ss. e p. 62).

8 Viola, F.; ZaCCARIA, G., Le ragioni del diritto, Il Mulino, Bologna, 2003, p. 172. 


\section{RAPPORTI DI EGUAGLIANZA O DI SUPREMAZIA/SUBORDINAZIONE}

Le due tentazioni riduzioniste (diritto è solo il diritto privato/diritto è solo il diritto pubblico) sono quindi da considerarsi ingiustificate e, al giorno d'oggi, persino inconcepibili. Peraltro, anche le dialettiche cittadino/straniero e Stato/straniero non possono essere declinate ora alla luce di una dimensione orizzontale (di eguaglianza) dei rapporti di diritto privato tra Stato, cittadini e stranieri, ora alla luce di una dimensione verticale (di supremazia/subordinazione) dei rapporti di diritto pubblico tra Stato, cittadini e stranieri. E ciò, non tanto perché nell'ineliminabile e potenziale conflittualità di rilievi privatistici e rilievi pubblicistici del diritto siano questi ultimi a dover prevalere in nome di un bene comune, quanto piuttosto per il fatto che gli uni e gli altri - a seconda dei livelli, degli strumenti, degli interessi in gioco - talvolta necessitano che ci si affidi alle forme naturali di comunità e all'autonomia personale di ciascuno, prima ancora che allo Stato, mentre, talaltra, esigono il rinvio al gruppo socio-culturale e allo Stato, prima ancora che all'individuo e alle sue relazioni interpersonali. È intuitivo del resto che alcune scelte personali abbiano significative conseguenze sotto il profilo dei rapporti interpersonali e sociali, ed è altrettanto intuitivo che le comunità e le istituzioni configurino un'idea di individuo conforme a certi valori, a determinati saperi e a particolari pratiche, intrecciando sfera personale e sfera sociale e orientandone atteggiamenti e comportamenti.

3.1. I costumi sono assai spesso il risultato del combinarsi di aspettative e di interessi, di timori e di giochi di forza, allo stesso modo lo sono anche gli istituti di cui un gruppo sociale e una comunità politica si dotano. Può accadere così che si sviluppino costumi originali, in nome di poteri e di privilegi, o magari per sfuggire ad abusi e a soprusi ${ }^{9}$, parimenti può accadere che un istituto consentito in alcune regioni, sia invece considerato illegale in altre.

È il caso del matrimonio poligamico, permesso nei paesi islamici e, al contrario, vietato negli altri. E proibito secondo il Comitato diritti umani ${ }^{10}$,

9 Si pensi ad esempio alle varie forme di transizione di genere che alcune culture conoscono (agli/ alle indiani/e Hijra e Sadhin, alle nordamericane Berdache, alle vergini giurate in Albania).

$10 \ll 24$. [...] States should provide information on these laws and practices and on the measures taken to abolish the laws and eradicate the practices which undermine the right of women to marry only when they have given free and full consent. It should also be noted that equality of treatment with regard to the right to marry implies that polygamy is incompatible with this principle. Polygamy violates the dignity of wom- 
perché la poligamia, nella sua manifestazione più diffusa, cioè quella poliginica, viola la dignità delle donne. Intanto però in molti Paesi - sia pure con diverse sfumature - le unioni poligamiche sono consentite, e in quei paesi in cui queste unioni sono considerate illegali si va diffondendo l'idea che in circostanze speciali (matrimoni celebrati all'estero e/o necessari per il benessere dei figli) esse possano essere riconosciute. D'altra parte, secondo qualche orientamento, l'unione poligamica potrebbe essere configurata come un diritto civile, diritto che in quanto tale non discriminerebbe alcuno visto che, solo per ragioni culturali, prevarrebbe la poliginia sulla poliandria.

$\grave{\mathrm{E}}$ ancora il caso del matrimonio precoce. Anche per questa tipologia di unione il Comitato diritti umani ha sottolineato come tra i fattori che compromettono la facoltà di decidere di sposarsi in piena libertà spicchi la minore età. Di qui la necessità per gli Stati di fissare un limite d'età al di sotto del quale non possa essere consentito contrarre matrimonio e che sia individuato sulla base di criteri eguali per entrambi i sessi ${ }^{11}$. Intanto però in molti Paesi, benché siano espressamente vietate, le unioni precoci sono comunque assai diffuse e qualche volta - laddove ricorrano taluni casi speciali (stupro, gravidanza) - sono persino consentite. In altri Paesi, poi, non è difficile immaginare la presenza di orientamenti con differenti origini che non disdegnino il ricorso alle unioni precoci, in quanto sarebbero capaci, grazie alla coincidenza di biologia-età della vita-procreazione, di avversare quel declino demografico che - diversamente dal resto del mondo - affligge qualche Paese europeo.

3.2. Che fare? Come conciliare le diverse posizioni? Chi ha ragione? $\mathrm{Si}$ può certo rispondere rinviando semplicemente agli ordinamenti, in nome della forza creatrice di chi pone e di chi attua il diritto (pubblico), così che nel sistema italiano tanto l'unione poligamica quanto l'unione precoce sono

en. It is an inadmissible discrimination against women. Consequently, it should be definitely abolished wherever it continues to exist» (General Comment No. 28: Equality of rights between men and women (article 3), adottato il 29 marzo 2000 in base all'art. 40, paragrafo 4, del Patto Internazionale sui diritti civili e politici).

11 «23. [...] Men and women have the right to enter into marriage only with their free and full consent, and States have an obligation to protect the enjoyment of this right on an equal basis. Many factors may prevent women from being able to make the decision to marry freely. One factor relates to the minimum age for marriage. That age should be set by the State on the basis of equal criteria for men and women. These criteria should ensure women's capacity to make an informed and uncoerced decision. A second factor in some States may be that either by statutory or customary law a guardian, who is generally male, consents to the marriage instead of the woman herself, thereby preventing women from exercising a free choice» (ivi). 
rifiutate anche dall'esplicita previsione secondo cui «1. Lo straniero può chiedere il ricongiungimento per i seguenti familiari: a) coniuge non legalmente separato e di età non inferiore ai diciotto anni; [...] d) genitori a carico, qualora non abbiano altri figli nel Paese di origine o di provenienza, ovvero genitori ultrasessantacinquenni, qualora gli altri figli siano impossibilitati al loro sostentamento per documentati, gravi motivi di salute. [...] 1-ter. Non è consentito il ricongiungimento dei familiari di cui alle lettere a) e d) del comma 1 , quando il familiare di cui si chiede il ricongiungimento è coniugato con un cittadino straniero regolarmente soggiornante con altro coniuge nel territorio nazionale» ${ }^{12}$.

Com'è intuitivo, il rinvio all'ordinamento, se, per un verso, confermerebbe il primato del diritto pubblico - e come detto la forza creatrice di chi pone la norma e di chi ne assicura la sua osservanza - per l'altro verso, però, lascerebbe inevasa la domanda: chi ha ragione? O per meglio dire: quale delle due prospettive (a favore della monogamia o invece della poligamia, a favore dei limiti d'età o invece del matrimonio precoce) può essere ritenuta conforme al diritto, inteso come attività che garantisce l'inclinazione naturale dell'uomo alla vita associata? E in tal senso, come attività che, anche nella sua dimensione di diritto pubblico, inevitabilmente nasce ed esplicita l'innata esigenza di socialità e di regolarità. Piuttosto che espressioni e meri precipitati della forza creatrice di un diritto ex nibilo, allora, le norme positive devono essere considerate come i prodotti di un'attività che permette e mantiene la vita associata.

Porsi la domanda: chi ha ragione? e per meglio dire, quale delle due prospettive (monogamia/poligamia, matrimonio maturo/matrimonio precoce) può essere ritenuta conforme al diritto, significa quindi comparare le diverse prospettive e valutare la possibilità che con l'una e con l'altra si realizzi, o meno, quell'innata tendenza alla socialità che è connaturata al diritto. Altrimenti quel che resta è un quadro normativo poco giustificato nella sua obbligatorietà e, per il nostro tema, non sufficientemente adeguato al crescente flusso migratorio e soprattutto non rispondente, nella legislazione, alla salvaguardia di quei diritti inalienabili e costitutivi della persona, che molti Stati hanno sottoscritto ed espressamente difeso nelle dichiarazioni internazionali, ma che non sempre trovano riscontro nella legislazione e nella prassi nazionale.

12 Art. 29, Testo unico delle disposizioni concernenti la disciplina dell'immigrazione e norme sulla condizione dello straniero (D.Lgs 286/1998, come modificato da ultimo dalle Leggi: 14 luglio 2017, n. 110, 7 aprile 2017, n. 47, 7 luglio 2016, n. 122, e dal D.Lgs. 29 ottobre 2016, n. 203). 


\section{DOVERE DI SOLIDARIETÀ E CITTADINANZA}

Nel sempre possibile contrasto fra autodeterminazione e sottomissione, identità personale e identità collettiva, privato e pubblico, è giocoforza necessario che si ritrovino delle idee e delle linee-guida che, oltre a giustificare l'accoglienza dello straniero, concorrano a distinguere tra loro i comportamenti irrilevanti, quelli permessi e quelli che, invece, devono essere vietati. Lidea di solidarietà è sotto questo aspetto particolarmente proficua, sia per il portato $(\mathrm{e}$ la forza: i socialmente fortunati vengono in aiuto dei meno fortunati) sia per i risvolti giuridici ${ }^{13}$.

4.1. Diritto-diritti-solidarietà: si pensi alla solidarietà attiva o passiva in caso di pluralità di creditori o di debitori in una stessa obbligazione; si pensi alla solidarietà sottesa al concetto di responsabilità oggettiva; si pensi ancora a quei sistemi di solidarietà che insieme all'uguaglianza costituiscono il fondamento della sicurezza sociale; si pensi inoltre alla solidarietà nazionale momento essenziale della cittadinanza politica (e anche di quella sociale ${ }^{14}$ ), come pure alla solidarietà civile (sulla base del volontariato e gestita da organizzazioni senza scopo di lucro); infine, anche se non da ultimo, si pensi alla solidarietà familiare.

Tutte queste forme di solidarietà ${ }^{15}$, che nell'ordinamento si influenzano a vicenda e che per certi versi si presuppongono l'un l'altra (ad es. nessun sistema

13 Si veda la ricostruzione di LOSANO, M. G., «La questione sociale e il solidarismo francese: attualità di una dottrina antica», Sociologia del diritto, 1, 2008, pp. 5-26, <http://www.dircost.unito. it/dizionario/pdf/Losano> (18 agosto 2017).

14 La cittadinanza sociale, distinta dalla cittadinanza politica, si basa su tre pilastri: la sicurezza sociale, i servizi pubblici e le libertà collettive garantite dal diritto del lavoro (libertà sindacale, contrattazione collettiva e diritto di sciopero). Questa cittadinanza sociale, che non si acquisisce per il fatto della nascita (ius sanguinis) o per il fatto di nascere nel territorio dello Stato (ius soli), unisce tutti coloro che contribuiscono alla solidarietà nazionale mediante tasse e contributi nel godimento dei servizi pubblici.

15 Sottolinea Supiot: «la solidarité se distingue aussi bien de l'assurance que de la charité. A la différence de l'assurance privée, qui s'appuie sur un calcul actuariel des risques (par une méthode statistique), un régime de solidarité repose sur l'appartenance à une communauté, qu'elle soit nationale, professionnelle ou familiale. Les membres de cette communauté qui sont à un moment donné les plus fortunés, ou les moins exposés au risque, contribuent davantage que les moins fortunés ou les plus exposés, mais tous ont les mêmes droits. A la différence de la charité (ou de son avatar contemporain, le care), la solidarité ne divise donc pas le monde entre ceux qui donnent et ceux qui reçoivent : tous doivent contribuer au régime selon leurs capacités, et tous ont le droit d'en bénéficier selon leurs besoins. Expression de l'égale dignité des êtres humains, l'organisation de la solidarité est un frein à l'extension de la logique marchande à 
previdenziale potrebbe resistere a lungo in caso di scomparsa della solidarietà familiare), hanno in comune la circostanza che non si lasciano appiattire e dissolvere in un puro calcolo di interessi, ma sono anzi fattori di resistenza alle leggi del mercato e della competizione economica ${ }^{16}$, perché operano con riferimento a un interesse politico che trascende gli elementi economici della società.

La nozione di solidarietà è inoltre il presupposto dello Stato sociale. Qui, infatti, al di là delle sue diverse versioni e formule politiche, viene prospettato in generale un diritto che rappresenta il medium attraverso il quale le varie esigenze socio-antropologiche possono strutturarsi storicamente e trovare risposta. In breve, il diritto sociale quale tecnica di umanizzazione che risponde all'esigenza di inclusione concreta, un'esigenza questa che diventa ancor più pressante visti gli sviluppi contemporanei della società dell'informazione e della globalizzazione.

E ancora: la nozione di solidarietà è il presupposto dell'Unione europea. Si pensi, ma solo a mo' d'esempio, alla regolazione del lavoro in Europa a fronte di trasformazioni economico-produttive che stanno alterando la capacità di protezione sociale delle tradizionali garanzie del lavoro fordista, al rapporto Supiot e ai cd. diritti di prelievo sociale ${ }^{17}$. Si tratta di riconoscere ai lavoratori periodi di aspettativa dal lavoro retribuito perché possano assumersi direttamente quelle attività sociali che sono produttive di una socialità migliore, ma che, ovviamente, richiedono tempo ed energie. Attività di cura, di formazione, di studio, impegni sociali, attività di volontariato, verrebbero così riconosciute e legittimate dal diritto come beni pubblici e, come tali, ne verrebbero tutelate e promosse l'attivazione e l'implementazione ${ }^{18}$.

Ed è sempre la nozione di solidarietà a costituire la chiave di lettura dell'integrazione europea, di quell'integrazione così poco reale vista le sue caratteristiche: integrazione negativa, piuttosto che positiva; decostruzione dei diritti sociali nazionali, anziché costruzione. «Abbiamo politiche dette di valorizzazione del capitale umano e che si esprimono attraverso la flex security [...].

toutes les activités humaines. C'est pourquoi elle est depuis trente ans la cible privilégiée des politiques néolibérales». SUPIOT, A., «Au fondement de la citoyenneté, ni assurance ni charité, la solidarité» Le monde diplomatique, 3 (novembre 2014), < https://www.monde-diplomatique.fr/2014/11/SUPIOT/50963>, 2014 (18 agosto 2017).

16 Ibidem.

17 SUPIOT, A.; BARBIERI, P. (a cura di), Il futuro del lavoro. Trasformazioni dell'occupazione e prospettive della regolazione del lavoro in Europa, trad. it., Carocci, Roma, 2003.

18 Anche se le sentenze: Viking (2007), Laval (2007) e Ruffert (2008), si sono decisamente discostate da simile orientamento. 
La concezione oggi dominante è che bisogna adattare gli uomini alle esigenze del mercato, e non adattare invece i mercati alle esigenze degli uomini. Questa concezione si sviluppa in una serie di categorie giuridiche che fioriscono, oggi, nei vari atti creati dall'Unione Europea, quando si parla di flessibilità e non di libertà, quando si parla di employability e non di capacità, quando si parla di capitale umano e non di capacità professionale. Quindi sempre ci sono le cose, i prodotti al centro dell'interesse, e non gli uomini ${ }^{19}$.

4.2. Secondo l'art. 2 della nostra Costituzione, la Repubblica, nel riconoscere i diritti inviolabili, richiede l'adempimento dei doveri inderogabili di solidarietà politica, economica e sociale. Com'è chiaro, con l'espressione diritti inviolabili si rinvia tanto ai diritti di libertà (e con questi al dovere di astensione da parte dello Stato e dei consociati), quanto ai diritti sociali (e con questi alla necessità che invece lo Stato intervenga così da superare alcune forme di privazione e di diseguaglianza). Proprio i diritti sociali, per la funzione svolta, hanno come immediato riferimento l'adempimento dei doveri di solidarietà, realizzabili solo entro una compiuta comunità politica. Con l'espressione, poi, doveri inderogabili si rinvia tanto ai doveri esplicitamente previsti dalla Costituzione (artt. 4, 32, 34, 48, 52, 53, 54), quanto ai doveri riconosciuti dalla Corte costituzionale o dal legislatore ordinario.

L'astratta uguaglianza formale è così mitigata dal compito, assegnato alla Repubblica, di rimuovere gli ostacoli al pieno sviluppo della persona (art. 3, comma 2) e d'altra parte il concetto (e il dovere) di solidarietà è strettamente legato all'idea di promozione della persona, in quanto modalità attraverso la quale essa viene inverata e realizzata. Diversa dall'assistenza e dalla beneficenza, separata dal volontariato, la solidarietà giuridica limita l'esercizio delle libertà economiche e al contempo fa sì che lo Stato possa agire al di fuori del mercato e disattendere le sue regole. A suo fondamento - ovvero per la concreta attuazione dei doveri di solidarietà, così che lo Stato abbia i mezzi - si ritrova l'art. 53, secondo cui «tutti sono tenuti a concorrere alle spese pubbliche in ragione della loro capacità contributiva. Il sistema tributario è informato a criteri di progressività».

Il dovere di solidarietà implica un certo modo di vedere il rapporto diritto-economia-politica e incide chiaramente sul concetto di cittadinanza, sia

19 Così Supiot, A., «Fermare la decostruzione dell'Europa», Centro Internazionale di Studi Sociali, <http://www.ciss.it/web/category/autore/alain-supiot>, 2009 (18 agosto 2017). 
perché ogni cittadino è potenzialmente responsabile della realizzazione dei bisogni altrui, sia perché - nel riconoscere a tutti i cittadini il diritto al lavoro e nel promuovere tutte le condizioni che rendano effettivo questo diritto (art. 4, comma 1) - «ogni cittadino inabile al lavoro e sprovvisto dei mezzi necessari per vivere ha diritto al mantenimento e all'assistenza sociale» (art. 38, comma 1).

I brevi cenni qui riferiti non possono trascurare il fatto che nelle società contemporanee l'idea di solidarietà deve misurarsi al contempo con molteplici livelli (tra gli Stati, tra le società, tra le generazioni) e con diverse dimensioni di tutela (ad esempio dei cittadini, dei cittadini comunitari dell'Unione Europea, dei cittadini di Paesi in via di sviluppo). Ma non è tutto, perché oggi la nozione di solidarietà è chiamata a misurarsi anche con i differenti compiti svolti ora dal sistema pubblico, ora dal sistema privato (sociale), il cui ruolo diventa via via più importante. $\grave{E}$ del tutto evidente, poi, che il tema e l'idea di solidarietà hanno a che fare pure con la tutela dei diritti fondamentali degli stranieri immigrati, così come con l'obiettivo di realizzare la più ampia coesione sociale cittadini-stranieri. Entro i confini europei, ma anche oltre, è così richiesta una nuova teoria della cittadinanza, che - nel rispetto dell'art. 78.1 del Trattato sul funzionamento dell'Unione Europea («L'Unione sviluppa una politica comune in materia di asilo, di protezione sussidiaria e di protezione temporanea, volta a offrire uno status appropriato a qualsiasi cittadino di un paese terzo che necessita di protezione internazionale e a garantire il rispetto del principio di non respingimento») e dell'art. 13.2 della Dichiarazione universale dei diritti dell'uomo («Ogni individuo ha diritto di lasciare qualsiasi Paese, incluso il proprio, e di ritornare nel proprio Paese») - sappia conciliare l'autodeterminazione dei popoli con il dovere di solidarietà e, per ciò stesso, con i diritti fondamentali della persona, quali: il diritto alla salute (ovvero il diritto di fruire di tutte le prestazioni indifferibili e urgenti $\left.\mathrm{i}^{20}\right)$; il diritto a condizioni di lavoro equivalenti a quelle dei

20 «Ai cittadini stranieri presenti sul territorio nazionale, non in regola con le norme relative all'ingresso ed al soggiorno, sono assicurate, nei presidi pubblici ed accreditati, le cure ambulatoriali ed ospedaliere urgenti o comunque essenziali, ancorché continuative, per malattia ed infortunio e sono estesi i programmi di medicina preventiva a salvaguardia della salute individuale e collettiva. Sono, in particolare garantiti: a) la tutela sociale della gravidanza e della maternità, a parità di trattamento con le cittadine italiane, ai sensi delle leggi 29 luglio 1975, n.405, e 22 maggio 1978, n. 194, e del decreto del Ministro della sanità 6 marzo 1995, pubblicato nella Gazzetta Ufficiale n.87 del 13 aprile 1995, a parità di trattamento con i cittadini italiani; b) la tutela della salute del minore in esecuzione della Convenzione sui diritti del fanciullo del 20 novembre 1989, ratificata e resa esecutiva ai sensi della legge 27 maggio 1991, n. 176; c) le vaccinazioni se- 
cittadini ${ }^{21}$; il diritto al ricongiungimento familiare; il diritto di partecipare alla vita pubblica; la tutela giurisdizionale dei diritti e degli interessi legittimi ${ }^{22}$; il diritto di prendere contatto con le autorità del Paese di cui si è cittadino.

4.3. Solidarietà (dovere di) e cittadinanza: senza la prima, la cittadinanza altro non è che un sistema di diritti esclusivo e privilegiato, un sistema obiettivamente odioso che per il suo forte presupposto - la comunità di storia e di destino - finisce col sottrarsi alla disciplina civica per divenire mero fattore di esclusione e di diseguaglianza. Con la prima, invece, la cittadinanza riconquista il proprium, il suo autentico portato e, cioè, la capacità di affermare e di promuovere i diritti umani ${ }^{23}$.

In particolare, con la solidarietà, l'istituto della cittadinanza può assumere un ruolo chiave nel contrastare il fenomeno della tratta degli esseri umani: un fenomeno, questo, che il più delle volte rappresenta il prodromo per la realizzazione di successive e ulteriori forme di assoggettamento (lavoro forzato, matrimonio forzato, prostituzione, pornografia, servitù domestica, traffico di organi...), forme di assoggettamento delle quali sono vittime per lo più le donne. Le convenzioni internazionali obbligano gli Stati ad adottare una serie di misure volte a prevenire e reprimere la tratta, quindi non si limitano a prevedere l'obbligo negativo di non commettere tratta degli esseri umani, ma prevedono anche quello positivo di adottare tutte le misure necessarie per proteggere le persone sottoposte alla loro giurisdizione. In quest'ottica, per le odierne migranti, e per le nuove schiave, l'istituto della cittadinanza po-

condo la normativa e nell'ambito di interventi di campagne di prevenzione collettiva autorizzati dalle regioni; d) gli interventi di profilassi internazionale; e) la profilassi, la diagnosi e la cura delle malattie infettive ed eventuale bonifica dei relativi focolai» (così l'art. 35.3 del già citato Testo unico delle disposizioni concernenti la disciplina dell'immigrazione e norme sulla condizione dello straniero).

21 E se si tratta di «stranieri titolari di carta di soggiorno o di permesso di soggiorno di durata non inferiore ad un anno», essi «sono equiparati ai cittadini italiani ai fini della fruizione delle provvidenze e delle prestazioni, anche economiche, di assistenza sociale, incluse quelle previste per coloro che sono affetti da morbo di Hansen o da tubercolosi, per i sordomuti, per i ciechi civili, per gli invalidi civili e per gli indigenti» (art. 41, ivi).

22 Che non si perde per il fatto di rimanere disoccupati: «la perdita del posto di lavoro non costituisce motivo di revoca del permesso di soggiorno al lavoratore extracomunitario ed ai suoi familiari legalmente soggiornanti» (art. 22.11, ivi), così che anche per loro continua a valere la garanzia del godimento dei «diritti in materia civile» e di «parità di trattamento e piena uguaglianza di diritti rispetto ai lavoratori italiani» (art. 2, commi 2 e 3, ivi).

23 Ho trattato l'argomento parecchio tempo fa nel mio «Desiderai essere un cittadino». Oltre il retaggio simbolico della moderna sovranità, Giappichelli, Torino, 1996. 
trebbe rivelarsi uno strumento idoneo alla repressione della tratta. In breve: da deportate, esuli e profughe, esse diverrebbero cittadine con pieni diritti e compiuta rappresentanza ${ }^{24}$.

\section{L'ATTENZIONE DEI TEORICI DEL DIRITTO PUBBLICO ALLA DOTTRINA SOCIALE DELla Chiesa. Tra libertà E BENE COMUNE}

Lidea e la pratica di solidarietà, nella loro dinamicità, richiedono continue riletture, rivisitazioni e anche contaminazioni. Se così, l'attenzione dei teorici del diritto pubblico alla dottrina sociale della Chiesa, oltre che auspicabile, si rivela senz'altro necessaria per fare avanzare i diritti umani - compresi naturalmente i diritti degli emigranti e dei rifugiati - affrontando con la dovuta serietà le condizioni politiche ed economiche che spingono le persone ad abbandonare i propri Paesi, così come il fenomeno della mobilità del capitale, che accelera le migrazioni e lo sfruttamento nel lavoro da parte dei Paesi ospitanti.

Dai concetti di libertà e di bene comune della dottrina sociale della Chiesa si traggono delle indicazioni e delle implicazioni essenziali. Si pensi alla libertà, al suo valore e ai suoi limiti: l'uomo può volgersi al bene soltanto nella libertà, in tal modo genera se stesso, è padre del proprio essere, costruisce l'ordine sociale. È chiaro però che questa libertà non è illimitata: deve arrestarsi di fronte all'albero della conoscenza del bene e del male, deve arrestarsi perché «non ha il suo punto di partenza assoluto e incondizionato in se stessa, ma nell'esistenza dentro cui si trova e che rappresenta per essa, nello stesso tempo, un limite e una possibilità». Un limite e una possibilità, il cui retto esercizio esige precise condizioni di ordine economico, sociale, giuridico, politico e culturale che troppo spesso invece sono misconosciute e violate ${ }^{25}$.

Di qui una duplice implicazione: nell'esercizio della libertà, l'uomo compie atti moralmente buoni, che, per un verso, sono costruttivi della sua persona

24 Cfr. il mio «Diritti dell'uomo/Diritti del cittadino», in Amato Mangiameli, A. C., Arte e/o tecnica. Sfide giuridiche, cit., pp. 173-183.

25 Pontificio Consiglio della Giustizia e della Pace, Compendio della Dottrina sociale della Chiesa, cap. III. La persona umana e i suoi diritti. III. La persona umana e i suoi molti profili; c) La libertà della persona (nn. 135-143); cap. IV I principi della dottrina sociale della Chiesa. VII I valori fondamentali della vita sociale; c) La libertà (nn. 199-200). <http://www.vatican.va/ roman_curia/pontifical_councils/justpeace/documents/rc_pc_justpeace_doc_20060526_compendio-dott-soc_it.html>, 2004 (18 agosto 2017). 
- che non può trovare compimento solo in se stessa, a prescindere cioè dal suo essere con e per gli altri - e che, per l'altro, sono produttivi della società, che non è semplice convivenza, ma ricerca senza posa (in forma pratica e non soltanto ideale) del bene: ovvero del senso e della verità rintracciabili nelle forme di vita sociale esistenti. Nessuna forma espressiva della socialità - dalla famiglia, al gruppo sociale intermedio, all'associazione, all'impresa di carattere economi$\mathrm{co}$, alla città, alla regione, allo Stato, fino alla comunità dei popoli e delle Nazioni - può eludere l'interrogativo circa il proprio bene comune, che è costitutivo del suo significato e autentica ragion d'essere della sua stessa sussistenza ${ }^{26}$.

Com'è intuitivo, le esigenze del bene comune derivano dalle condizioni sociali di ogni epoca e sono strettamente connesse al rispetto e alla promozione integrale della persona e dei suoi diritti fondamentali. Concretamente tali esigenze riguardano anzitutto la pace, la cooperazione internazionale, la salvaguardia dell'ambiente, l'impegno per un'organizzazione dei poteri dello Stato e per un ordinamento giuridico in vista del bene comune, la prestazione di quei servizi essenziali delle persone (alimentazione, abitazione, salute, lavoro, educazione, libera circolazione delle informazioni, accesso alla cultura, tutela della libertà religiosa) ${ }^{27}$.

5.1. Le ulteriori e importanti considerazioni sono: a) il bene comune, che impegna tutti ed è arduo da raggiungere, è conseguente alle più elevate inclinazioni dell'uomo, perché richiede la capacità e la ricerca costante del bene altrui come se fosse proprio; b) la responsabilità di conseguire il bene comune compete, oltre che alle singole persone, anche allo Stato, poiché il bene comune è la ragion d'essere dell'autorità politica.

Lo Stato, infatti, deve garantire coesione, unitarietà e organizzazione alla società civile di cui è espressione, in modo che il bene comune possa essere conseguito con il contributo di tutti i cittadini. L'uomo singolo, la famiglia, i corpi intermedi non sono in grado di pervenire da se stessi al loro pieno svi-

26 «Il bene comune della società non è un fine a sé stante; esso ba valore solo in riferimento al raggiungimento dei fini ultimi della persona e al bene comune universale dell'intera creazione. Dio è il fine ultimo delle sue creature e per nessun motivo si può privare il bene comune della sua dimensione trascendente, che eccede ma anche dà compimento a quella storica. [...] Una visione puramente storica e materialistica finirebbe per trasformare il bene comune in semplice benessere socio-economico, privo di ogni finalizzazione trascendente ovvero della sua più profonda ragion d'essere» (Ivi, cap. IV I principi della Dottrina sociale della Chiesa; II Il principio del bene comune, n. 170).

27 Ivi, nn. 164-170. 
luppo; da ciò deriva la necessità di istituzioni politiche, la cui finalità è quella di rendere accessibili alle persone i beni necessari (materiali, culturali, morali, spirituali) per condurre una vita veramente umana. Il fine della vita sociale è il bene comune storicamente realizzabile.

Per assicurare il bene comune, il potere pubblico ha il compito specifico di armonizzare con giustizia i diversi interessi settoriali, ha la responsabilità di conciliare con correttezza i beni particolari di gruppi e di individui, ed è tenuto ad interpretare il bene comune non soltanto secondo gli orientamenti della maggioranza, ma nella prospettiva del bene effettivo di tutti i membri della comunità civile, compresi quelli in posizione di minoranza.

Così, per assicurare il bene comune, bisogna che la convivenza civile e politica conquisti il suo significato più profondo, ovvero compia il passaggio dal campo del diritto -che «è quello dell'interesse tutelato e del rispetto esteriore, della protezione dei beni materiali e della loro ripartizione secondo regole stabilite» - al campo dell'amicizia - che è invece «quello del disinteresse, del distacco dai beni materiali, della loro donazione, della disponibilità interiore alle esigenze dell'altro. L'amicizia civile, così intesa, è l'attuazione più autentica del principio di fraternità, che è inseparabile da quello di libertà e di uguaglianza. Si tratta di un principio rimasto in gran parte non attuato nelle società politiche moderne e contemporanee, soprattutto a causa dell'influsso esercitato dalle ideologie individualistiche e collettivistiche $\gg^{28}$.

\section{UNA DIALETTICA DIVERSA: DOVERI, PIUTTOSTO CHE DIRITTI}

Libertà (dignità, uguaglianza) e bene comune. C'è un termine che meglio di altri può costituire una sorta di trattino di congiunzione (di segno/simbolo ortografico) tra libertà-dignità-uguaglianza-bene comune. Ł̀ il termine dovere.

La cittadinanza rispecchia prima di tutto un ordine e il cittadino (della $\pi o ́ \lambda \imath \varsigma$, come pure dello Stato-nazione) non deve essere pensato a partire dalla categoria diritto (soggettivo), bensì dal presupposto del dovere. Egli, in altre parole, può dirsi tale solo - e soltanto - nei termini in cui la sua azione rappresenti il portato di un'obbligazione etico-giuridica. A differenza dello straniero - che versa in uno stato di soggezione e si trova in una posizione negativa-passiva (poiché gli viene richiesto di non attivarsi e, più in generale,

28 Ivi, cap. VIII La comunità politica; II Il fondamento e il fine della comunità politica, n. 390. 
di non superare certi limiti) - il cittadino ha dei doveri inderogabili che richiedono l'adempimento e che, perciò, presuppongono un comportamento attivo da parte del soggetto. Sotto questo profilo, un classico esempio è fornito dal dovere di fedeltà alla Repubblica. Ovviamente, il significato di questo dovere emerge, non nel momento in cui l'autorità dello Stato è forte e indiscussa, ma allorquando la crisi delle istituzioni è tale da non rendere utilizzabile ogni altro strumento giuridico (coercitivo).

Doveri: se si riconosce l'antecedenza ai doveri, piuttosto che ai diritti, ci si sbarazza dei comitati di cittadini (intolleranti e xenofobi) e si riabilita il cittadino della comunità statuale (vale a dire di quella comunità volta a sviluppare il principio di sussidiarietà). Svolta in questa direzione, poi, la riflessione consente di mettere a fuoco la continuità che esiste fra le istanze del cittadino e del civis mundi (e, quindi, dell'uomo tout court). Il cittadino deve fedeltà; il rifugiato politico e il rifugiato cosiddetto economico devono amicizia. Infine, il cittadino della comunità universale - sia pure in nome di un imperfetto diritto e di imperfette istituzioni - deve lealtà. Tali obblighi sono in rapporto di reciproca interdipendenza e fondazione, ovvero subordinati a un dovere giuridico-morale radicale e iniziale: impedire le violazioni dei diritti dell'uomo e dei popoli, osservando le norme di diritto internazionale.

Si tratta allora di mantenere intrecciate le due dimensioni del particolare e dell'universale. Un'espressione sintetica e declinata «in negativo» può rivelarsi qui un appropriato elemento di sostegno: né Caino né Abele. La protezione e la tutela del cittadino e del civis mundi richiedono, da un lato, il superamento di quel carattere usurpatorio e violento dell'uomo di città, e, dall'altro, la rimozione di quel carattere che, sprovvisto di radicamenti, è percepito solo come effimero fervore dell'uomo senza città. Quando, infatti, il cittadino si trasforma in Caino, «si rifiuta alla vita del nomade temendone l'infelicità $»^{29}$, non per questo smantella la curiosa dualità con se stessi: il volto dello straniero si rivela innanzitutto in noi stessi. Quando il civis mundi, l'apolide, il nomade, diventano Abele non per questo diventano subito migliori. Anche per l'estraneo (lo straniero) valgono necessariamente i tratti e le finalità, al di là delle ambiguità, dello spirito cittadino: garantire permanenza, sicurezza, durevolezza, senza le quali il mondo cittadino e il mondo in generale non sarebbero possibili.

Doveri concreti, per gli uni e per gli altri. Così, se da un lato bisogna che i cittadini (e con essi le istituzioni) prestino grande attenzione alla «lingua

29 D’Agostino, F., Per un'archeologia del diritto. Miti giuridici greci, Giuffrè, Milano, 1979, p. 148. 
materna dei migranti, attraverso la quale essi esprimono la mentalità, le forme di pensiero e di cultura ed i caratteri stessi della loro vita spirituale e delle tradizioni delle loro Chiese di origine» ${ }^{30}$, dall'altro, bisogna che chiunque si rechi presso un altro popolo, «deve fare molta stima del suo patrimonio, della sua lingua e dei suoi costumi [...] perciò i migranti si adattino volentieri alla comunità che li accoglie e si affrettino a impararne la lingua, cosicché se la permanenza si fa prolungata o diventa definitiva, possano più facilmente integrarsi nella nuova società ${ }^{31}$.

30 Pontificio Consiglio della Pastorale Per i Migranti e gli Itineranti, Istruzione Erga migrantes caritas Christi, Parte II Migranti e pastorale d'accoglienza, n. 38, <http://www.vatican. va/roman_curia/pontifical_councils/migrants/documents/rc_pc_migrants_doc_20040514_erga-migrantes-caritas-christi_it.html>, 2004 (18 agosto 2017).

31 Doveri già affermati nell'Instructio De pastorali migratorum cura, Caput I, De principiis generalibus, 10 De officiis erga communitatem excipientem, <http://w2.vatican.va/content/paul-vi/ it/motu_proprio/documents/hf_p-vi_motu-proprio_19690815_pastoralis-migratorum-cura. html>, 1969 (18 agosto 2017). 